
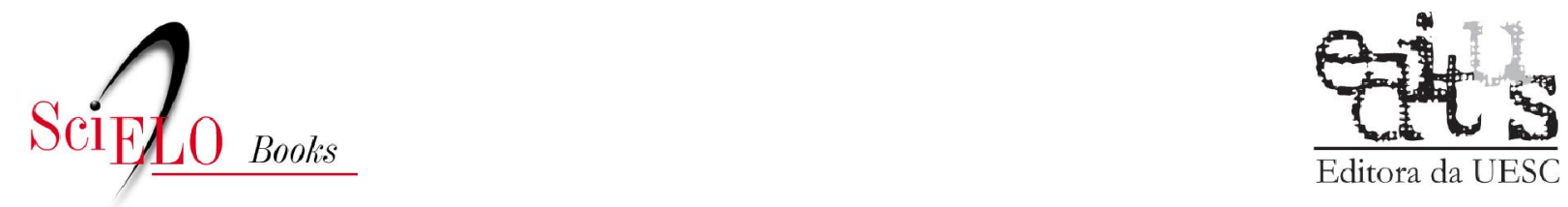

Editora da UESC

\title{
2 - A subfamília Amblyoponinae na Região Neotropical
}

\author{
Flavia A. Esteves \\ Brian L. Fisher
}

ESTEVES, FA., and FISHER, BL. A subfamília Amblyoponinae na Região Neotropical. In:

DELABIE, JHC., et al., orgs. As formigas poneromorfas do Brasil [online]. Ilhéus, BA: Editus, 2015,

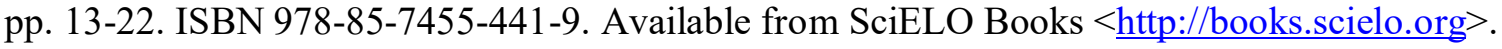

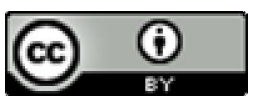

All the contents of this work, except where otherwise noted, is licensed under a Creative Commons Attribution 4.0 International license.

Todo o conteúdo deste trabalho, exceto quando houver ressalva, é publicado sob a licença Creative Commons Atribição 4.0.

Todo el contenido de esta obra, excepto donde se indique lo contrario, está bajo licencia de la licencia Creative Commons Reconocimento 4.0. 


\section{A subfamília Amblyoponinae na Região Neotropical}

Flavia A. Esteves, Brian L. Fisher

\section{Resumo}

A subfamília Amblyoponinae apresenta distribuição global e na região Neotropical inclui os gêneros Paraprionopelta Kusnezov, Prionopelta Mayr e Stigmatomma Roger.

Neste capítulo, apresentamos uma introdução à esta subfamília, destacando alguns aspectos de sua sistemática. Fornecemos informações sobre distribuição e biologia, e diagnoses para fêmeas e machos dos gêneros de Amblyoponinae de distribuição Neotropical. Incluímos imagens de alta resolução para ilustrar os gêneros que ocorrem nesta região biogeográfica.

Finalizamos o capítulo ressaltando alguns empecilhos para um maior conhecimento

da biologia e taxonomia da subfamília na região Neotropical. Infelizmente, todos os três gêneros presentes nesta região possuem problemas com relação à delimitação de suas espécies. Paraprionopelta é apenas conhecida por machos; Prionopelta amabilis, $P$. antillana e $P$. marthae não podem ser claramente identificadas com os caracteres morfológicos atualmente disponíveis; e a maioria das espécies de Stigmatomma precisa de redescrições mais detalhadas para que caracteres ambíguos, e amplamente utilizados atualmente, sejam substituídos por outros de maior valor diagnóstico. Por fim, apresentamos soluções para que estes problemas sejam superados.

ESTEVES, Flavia A.; FISHER, Brian L. A subfamília Amblyoponinae na região Neotropical. In: DELABIE, Jacques H. C. et al. As formigas poneromorfas do Brasil. Ilhéus: Editus, 2015. p. 13-22. 


\section{Abstract}

The subfamily Amblyoponinae in the Neotropical Region - The subfamily Amblyoponinae is widespread around the globe, and in the Neotropical bioregion includes the genera Paraprionopelta Kusnezov, Prionopelta Mayr and Stigmatomma Roger.

In this chapter, we offer an overview of the subfamily and highlight aspects of its systematics; and provide information on biology, distribution, and diagnosis for females and males of Amblyoponinae genera distributed in the Neotropics. We also included high-resolution images to illustrate the genera that occur in that bioregion.
We finalize this chapter by highlighting some issues for a comprehensive knowledge of the subfamily's biology and taxonomy in the Neotropical region. Unfortunately, all the three genera possess problems related to the identification of their species. Paraprionopelta is just known by the male caste; Prionopelta amabilis, P. antillana and $P$. marthae cannot be clearly called apart with the morphological characters currently available; and the majority of Stigmatomma species need more detailed descriptions, so that ambiguous characters, currently broadly used, may be replaced by others with higher diagnostic value. Finally, we present solutions to overcome these issues.

\section{Introdução}

A subfamília Amblyoponinae se distribui globalmente e atualmente agrupa os gêneros: Adetomyrma Ward (1994: 160); Amblyopone Erichson (1842: 260); Apomyrma Brown, Gotwald e Lévieux (1971: 259); Bannapone Xu (2000: 299); Concoctio Brown (1974: 29); Myopopone Roger (1861: 49); Mystrium Roger (1862: 245); Onychomyrmex Emery (1895: 349); Opamyrma Yamane, Bui e Eguchi $(2008,56)$; Paraprionopelta Kusnezov (1955: 270); Prionopelta Mayr (1866: 503); Stigmatomma Roger (1859: 250); Xymmer Santschi (1914: 311); além do gênero fóssil Casaleia Pagliano e Scaramozzino (1990: 5). Atualmente são reconhecidas mais de 130 espécies para a subfamília (BOLTON, 2014).

$\mathrm{O}$ velho mundo possui a maior diversidade genérica de Amblyoponinae. A região Indomalaia é a mais diversa, com sete gêneros (Bannapone, Myopopone, Mystrium, Opamyrma, Prionopelta, Stigmatomma e Xymmer), seguida pelas regiões Australiana e Afrotropical, ambas com seis gêneros (Amblyopone, Myopopone, Mystrium, Onychomyrmex, Prionopelta e Stigmatomma; Apomyrma, Concoctio, Mystrium, Prionopelta, Stigmatommae Xymmer, respectivamente).A região Neotropical possui 3 gêneros: Paraprionopelta, Stigmatomma e Prionopelta (ANTWEB, 2014a; BOLTON, 2014).

\section{Sistemática}

A relação de parentesco entre as linhagens de formigas com ramificação mais antiga, incluindo Amblyoponinae, ainda é incerta (BRADY et al., 2006; KÜCK et al., 2011; MOREAU; BELL, 2013), limitando nossa compreensão sobre os fatores que moldaram a evolução das formigas, e nossa habilidade para testá-los (WILSON e HÖLLDOBLER, 2005; LUCKY et al., 2013; MOREAU; BELL, 2013). Felizmente, o nosso conhecimento sobre a biologia evolutiva de formigas aumentou muito ao longo da última década (BOLTON, 2003; BRADY et al., 2006; MOREAU et al., 2006; RABELING et al., 2008; MOREAU, 2009; KÜCK et al., 2011; MOREAU; BELL, 2013; BRADY et al., 2014; WARD et al., 2014), e um dos resultados é que Amblyoponinae voltou a ser reconhecida como subfamília (BOLTON, 2003).

A relação filogenética entre os gêneros de Amblyoponinae também não está perfeitamente resolvida, mas caminhamos muito nesta direção. A subfamília aparentemente é formada por dois clados (BRADY et al.,2006; OULLETTE et al.,2006; SAUX et al., 2004): (1) Adetomyrma + Bannapone, Myopopone + Mystrium + Paraprionopelta + Stigmatomma + Xymmer; e (2) Amblyopone + Concoctio + Onychomyrmex + Prionopelta (YOSHIMURA e FISHER, 2012; FISHER, B.L., dados ainda não publicados); a posição de 
Apomyrma e Opamyrma não é conhecida. A delimitação dos gêneros dessa subfamília também está passando por transformações - Stigmatomma e Xymmer são aceitos novamente como gêneros depois de um longo tempo sinomizados em Amblyopone (YOSHIMURA; FISHER, 2012), e dados preliminares indicam a polifilia de Stigmatomma (FISHER, B.L., dados ainda não publicados).

\section{Gêneros de Amblyoponinae da Região neotropical}

\section{Paraprionopelta Kusnezov, 1955}

Paraprionopelta é conhecida por apenas uma espécie, Paraprionopelta minima Kusnezov (1955: 271), baseada na descrição de machos coletados na região de Tucumã, Argentina.

Os espécimes deste gênero foram coletados em diferentes ocasiões em Tucumã, Argentina, através de armadilhas luminosas, e assim, nada se sabe sobre sua biologia. Desde então, machos de Amblyoponinae que possuem caracteres semelhantes aos de Paraprionopelta foram coletados na Nicarágua, e suas imagens estão disponíveis em Antweb.org (2014b; CASENT0628950).

\section{Diagnose}

Mandíbulas com um dente. Antena com 10 segmentos (Figura 2-1A). Fórmula palpal 2:2 (2 maxilares e dois labiais) (KUSNEZOV, 1955). Cabeça oblonga (Figura 2-1A). Notaulus ausente ou fracamente desenvolvido. Pigóstilos presentes.

\section{Prionopelta Mayr, 1866}

Prionopelta possui atualmente 21 espécies válidas no mundo, das quais 5 ocorrem na região Neotropical: P. amabilis Borgmeier (1949: 203); P. antillana Forel (1909: 239); P. marthae Forel (1909: 240); P. modesta Forel (1909: 241); e P. punctulata Mayr (1866: 505).

Este gênero de formigas geralmente ocorre em habitats de florestas úmidas e nidifica dentro de troncos podres sobre o solo, nas camadas superficiais do solo, em frutos podres e secos sobre o solo, e no interior do solo.

O conhecimento atual sobre o comportamento de Prionopelta é derivado basicamente de três estudos baseados em observações de colônias cativas de P. amabilis (HOLLDOBLER; WILSON, 1986; HOLLDOBLER et al., 1992) e P. kraepelini Forel (1905: 3) (ITO; BILLEN, 1998). Em P. amabilis,

Figura 2-1 - Parátipo de Paraprionopelta minima (CASENT0172797): (A) vista dorsal da cabeça e (B) lateral do - corpo. Imagens: April Nobile; disponível em AntWeb.org.

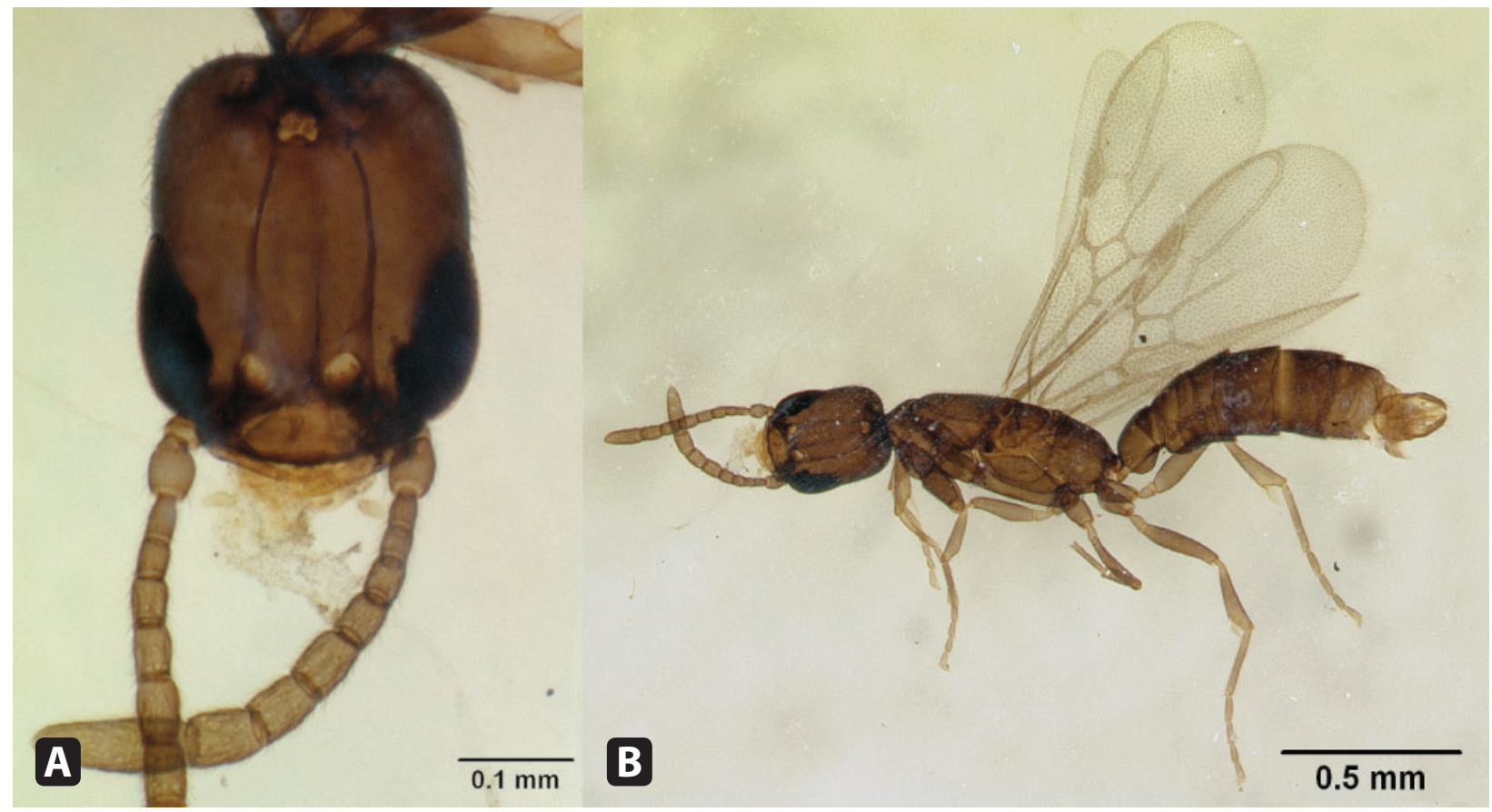


as colônias são polidômicas (também descrito para P. modesta; BROWN, 1960) e podem conter mais de 700 operárias e uma ou duas rainhas. Aparentemente as colônias de $P$. kraepelini são monogínicas. Todas as operárias de $P$. amabilis parecem ser capazes de botar ovos tróficos, os quais podem ser a fonte principal de alimento da rainha (esta se alimenta também de hemolinfa larval em $P$. kraepelini).

Aparentemente, o recrutamento em $P$. amabilis é feito através de vibrações corporais e secreções de uma glândula exócrina localizada no basitarso posterior; as operárias atacam presas vivas e as transportam de volta para a colônia, onde as larvas de alimentam diremente sobre elas (também observado em P. modesta; BROWN, 1960). Em cativeiro, P. amabilis prefere se alimentar de Campodeidae (Diplura), apesar de também aceitar centípedes geofilomorfos e outros poucos artrópodos. Operárias mais novas desta espécie cuidam principalmente dos imaturos, enquanto operárias mais velhas podem cuidar dos imaturos, da rainha, além de serem reponsáveis pelo forrageamento. Fragmentos do casulo das pupas são usados para forrar a câmera ocupada pelas pupas, provavelmente para mantê-la seca. Trofalaxia e transporte de alimento entre adultos de
P. amabilis nunca foi observado, e aparentemente não existe ferormônio de alarme.

\section{Diagnose}

Fêmeas (Figura 2-2): Mandíbula estreita e curta, subtriangular, armada com 3 dentes (Figura 2-2A). Margem anterior do clípeo convexa ou moderadamente projetada, armada com pequenas setas dentiformes (Figura 2-2A). Olhos compostos reduzidos nas operárias, localizados posteriormente à linha média da cabeça. Antena com 12 segmentos, raramente com 11; três ou quatro segmentos apicais alargados e alongados (Figura 2-2A). Fórmula palpal 2:2 (2 maxilares e 2 labiais) em todas as castas (BROWN, 1960). Espécimes geralmente pequenos e despigmentados.

Machos (Figura 2-3): Antenas com 13 segmentos; escapos curtos. Mandíbulas com dois dentes. Notaulus desenvolvido. Cor mais escura que a das fêmeas.

\section{Identificação das espécies neotropicais}

A chave mais atual disponível para identificação das espécies de Prionopelta da região

FIGURA 2-2 - Prionopelta amabilis. Operária (CASENT0260460): (A) vista dorsal da cabeça, (B) dorsal do corpo e (C) lateral do corpo. Imagens: Will Ericson; disponível em AntWeb.org.

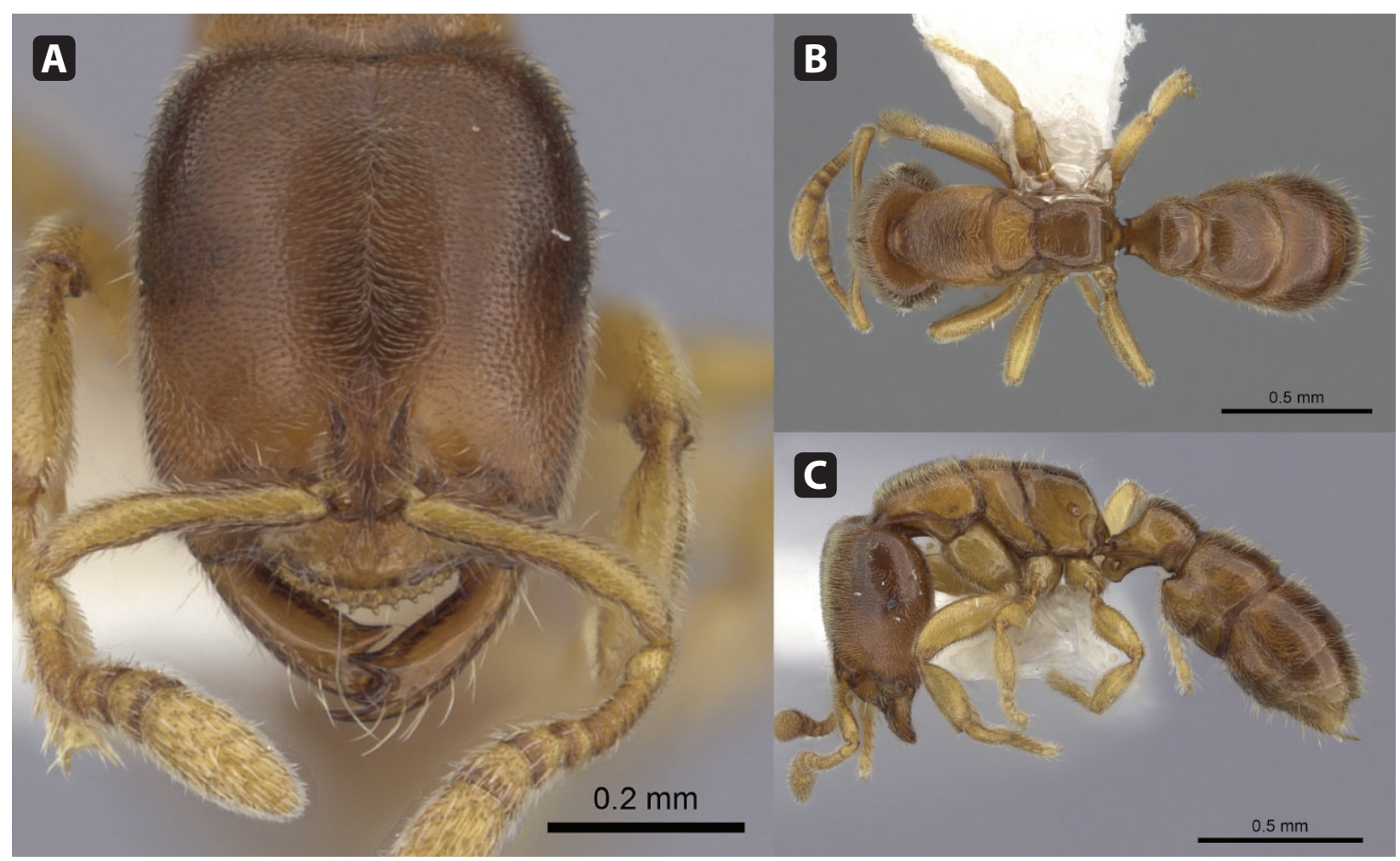


FIGURA 2-3 - Macho de Prionopelta punctulata (CASENT0173508). A: vista lateral do corpo; B: ápice do gáster retratando telômeros, aedeagus e pigóstilos (indicado pela seta); C: vista dorsal do corpo; (D) asas. Imagens: April Nobile; disponível em AntWeb.org.

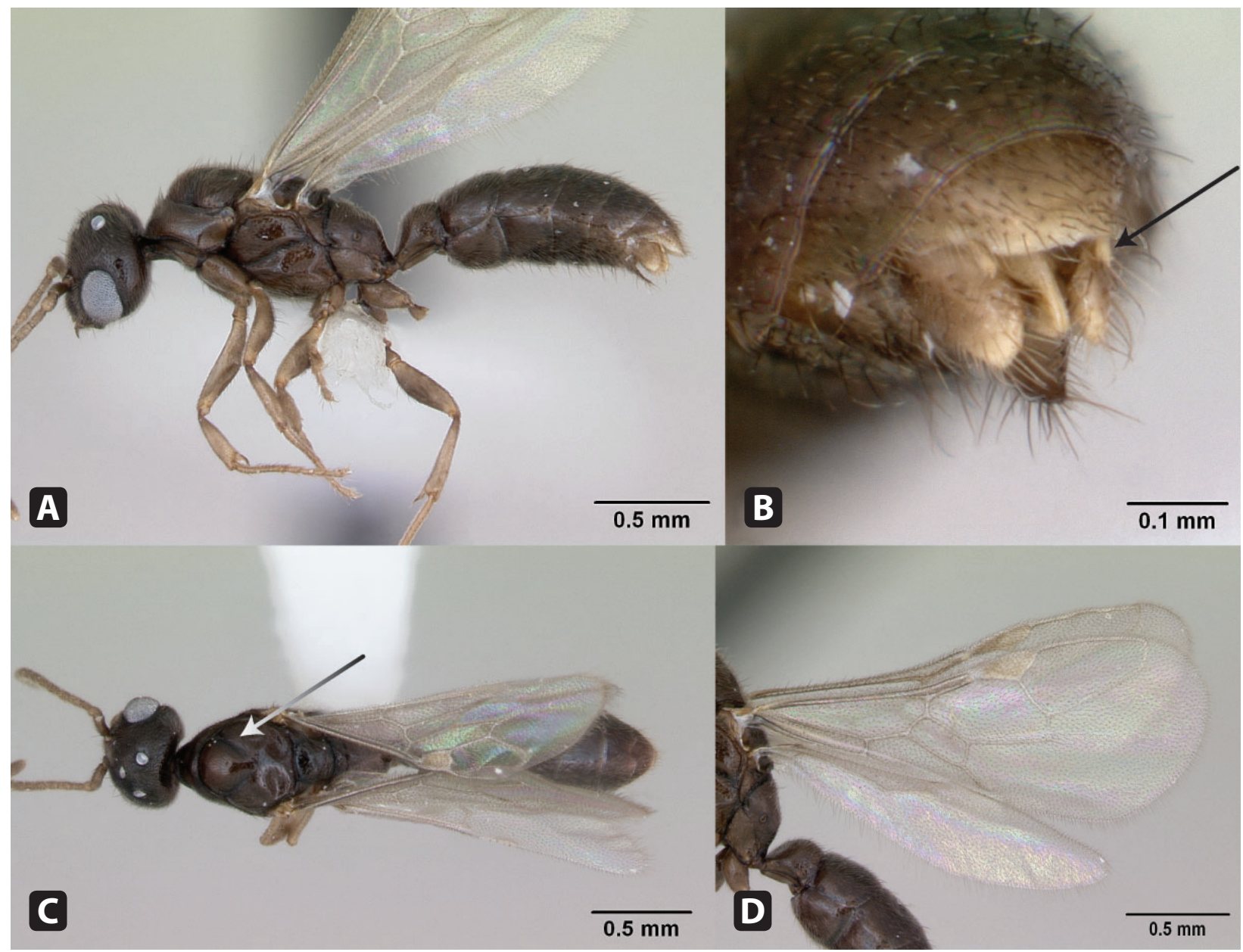

Neotropical (ARIAS-PENNA, 2008; modificada a partir de BROWN, 1960) possui alguns problemas: ignora a existência de $P$. marthae, nome atualmente válido (BROWN, 1965; BOLTON, 2014); e utiliza localização geográfica para diferenciar $P$. amabilis de P. antillana, que, no entanto, ocorrem em simpatria.

Uma nova chave baseada nas descrições existentes para as espécies e nas imagens dos espécimes tipo, disponíveis em Antweb.org, será em breve publicada (ESTEVES; FISHER, em preparação).

\section{Stigmatomma Roger, 1859}

Stigmatomma possui atualmente 67 espécies válidas no mundo, além de duas espécies fósseis. Na região Neotropical são encontradas 14 espécies: S. agostii (Lacau e Delabie, 2002: 36); S. armigerum (Mayr, 1887: 547); S. bierigi Santschi (1930: 75); S. chilense (Mayr, 1887: 547); S. cleae (Lacau e Delabie, 2002: 34); S. degeneratum (Borgmeier, 1957: 111);
S. egregium (Kusnezov, 1955: 274); S. elongatum Santschi (1912: 519); S. falcatum (Lattke, 1991: 1); S. heraldoi (Lacau e Delabie, 2002: 37); S. lurilabes (Lattke, 1991: 2); S. monrosi (Brown, 1960: 188); S. mystriops (Brown, 1960: 185); e S. orizabanum (Brown, 1960: 190).

Este é o gênero mais diverso de Amblyoponinae (BOLTON, 2014). Possui distribuição global, e seus representantes são geralmente encontrados em hábitats úmidos, nidificando dentro de troncos podres sobre o solo e no interior do solo. Stigmatomma pluto, por exemplo, nidifica entre 10 e $30 \mathrm{~cm}$ de profundidade no solo; o ninho é formado por câmeras pequenas (altura máxima de $3 \mathrm{~cm}$ ) (GOTWALD; LÉVIEUX, 1972).

Assim como outras Amblyoponinae, os membros de Stigmatomma são predadores especializados de outros artrópodes, especialmente quilópodos geofilomorfos (BROWN, 1960; GOTWALD; LÉVIEUX, 1972) - observações indicam que até $80 \%$ da dieta de $S$. silvestrii é composta por 
estes quilópodos (MASUKO, 1993), por exemplo. Em colônias cativas desta espécie, a operária aborda a presa com as mandíbulas abertas a um ângulo de $70-80^{\circ}$, e quando o par de setas longas e flexíveis, localizadas na margem anterior do clípeo, toca o corpo da presa, as mandíbulas se fecham com um único e rápido golpe contra a vítima (MASUKO, 1993).

Operárias de Stigmatomma são consideradas caçadoras solitárias (TRANIELLO, 1978; MASUKO, 1993), no entanto, existem registros de recrutamento para S. reclinatum (ITO, 1993a; BILLEN et al., 2005). Nesta espécie, quando uma operária subjuga uma presa grande, outras operárias são recrutadas (ITO, 1993a) através de uma trilha de feromônios excretados por uma glândula epitelial presente na face dorsal do pretarso da perna posterior (BILLEN et al., 2005).

Aparentemente colônias de algumas espécies de Stigmatomma são constantemente movidas para que as larvas se alimentem diretamente de presas grandes, que por causa do tamanho, inviabilizam seu transporte para o ninho (BROWN, 1960; MASUKO, 1993). No entanto, informações mais detalhadas sobre esse comportamento são escassas, provavelmente devido ao hábito hipogeico das espécies do gênero.
A maioria das rainhas imaturas possui asas. No entanto, existem registros para espécies de Stigmatomma do grupo reclinatum (região Indomalaia) de colônias sem rainhas distintas morfologicamente, onde a reprodução acontece através de gamergates (ITO, 1991, 1993b). Estas colônias poligínicas se organizam principalmente através de interações agressivas de dominância e interações químicas (ITO, 1993b). Além disso, intercastas intermediárias entre operárias e rainhas são encontradas em pelo menos uma espécie de Stigmatomma em Madagascar (observações pessoais).

\section{Diagnose}

Fêmeas (Figura 2-4): mandíbulas alongadas e lineares; ápice formado por um dente agudo; margens basal e mastigatória indistintas (aqui chamada de margem baso-mastigatória); margem baso-mastigatória com numerosos dentes, distribuídos em duas fileiras (Figura 2-4A). Porção mediana da margem anterior do clípeo geralmente convexa e projetada anteriormente; armada com setas dentiformes, associadas ou não basalmente a projeções cuticulares em forma de tubérculos (Figura 2-4A). Dente gular presente

FIGURA 2-4 - Holótipo de Stigmatomma armigerum. Operária (CASENT0915647): (A) vista dorsal da cabeça, (B) dorsal do corpo e (C) lateral do corpo. Imagens: Harald Bruckner; disponível em AntWeb.org.

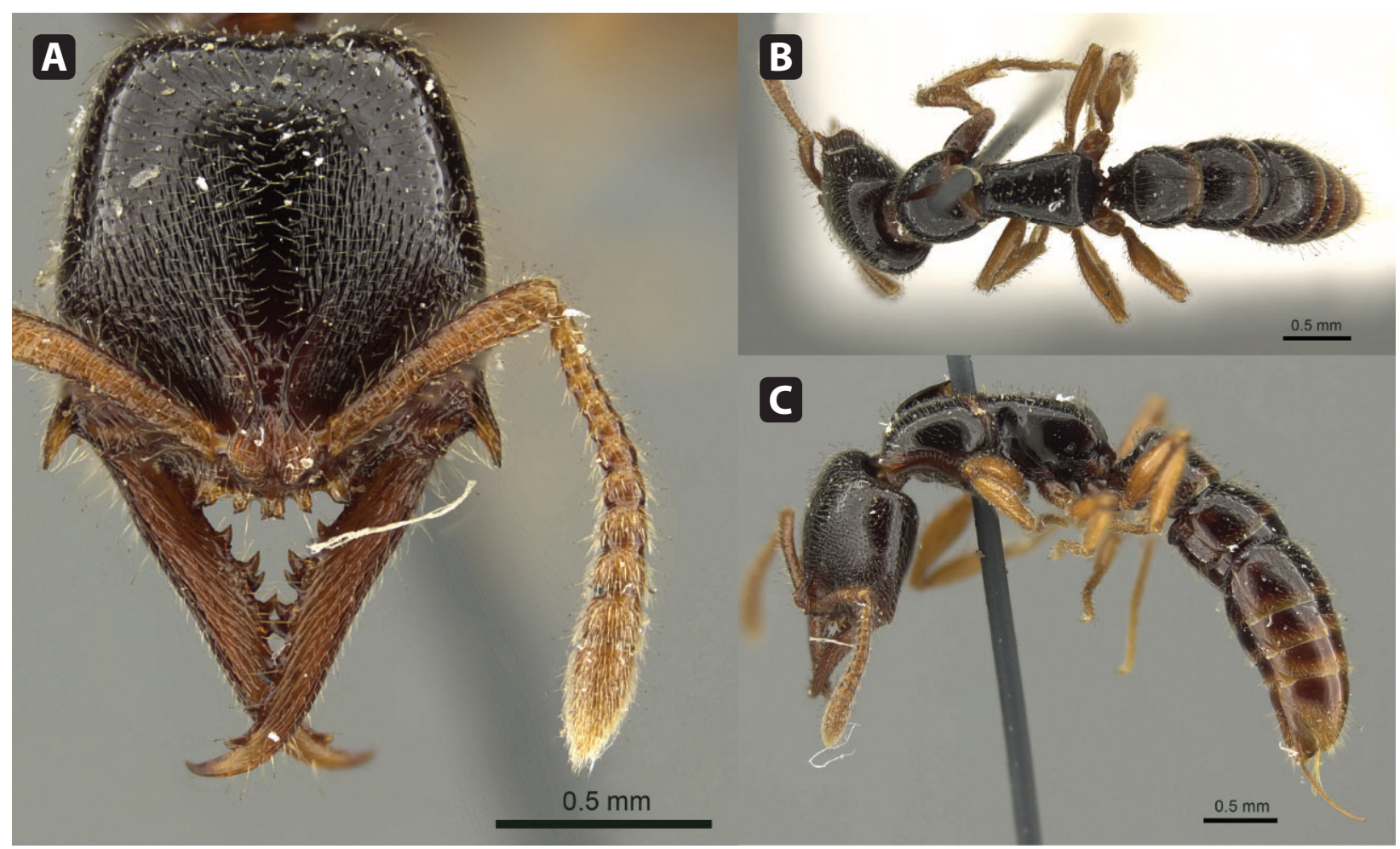


ou ausente. Processo torular fundido com lóbulos frontais em diversos níveis. Olhos compostos, quando presentes nas operárias, localizados posteriormente à linha média da cabeça. Segmentos antenais variando de 6 a 12. Fórmula palpal varia de 5:3 (5 maxilares e 3 labiais) a 1:2 (BROWN, 1960). Hipopígio (sétimo esterno abdominal) armado apicalmente com setas espiniformes em algumas espécies (LACAU; DELABIE, 2002; YOSHIMURA; FISHER, 2014). Ferrão bem desenvolvido e totalmente funcional.

Machos (Figura 2-5): mandíbulas com 1 dente apical (KUSNEZOV, 1955; YOSHIMURA; FISHER, 2014; observação pessoal). Antenas com 13 segmentos (KUSNEZOV, 1955). Escapos antenais reduzidos. Notaulus geralmente bem desenvolvido. Pigóstilos presentes.

FIGURA 2-5 - Macho de Stigmatomma egregium: vista dorsal da cabeça. Ilustração adaptada a partir de Kusnezov (1955: 274; figura 6) por F.A. Esteves.

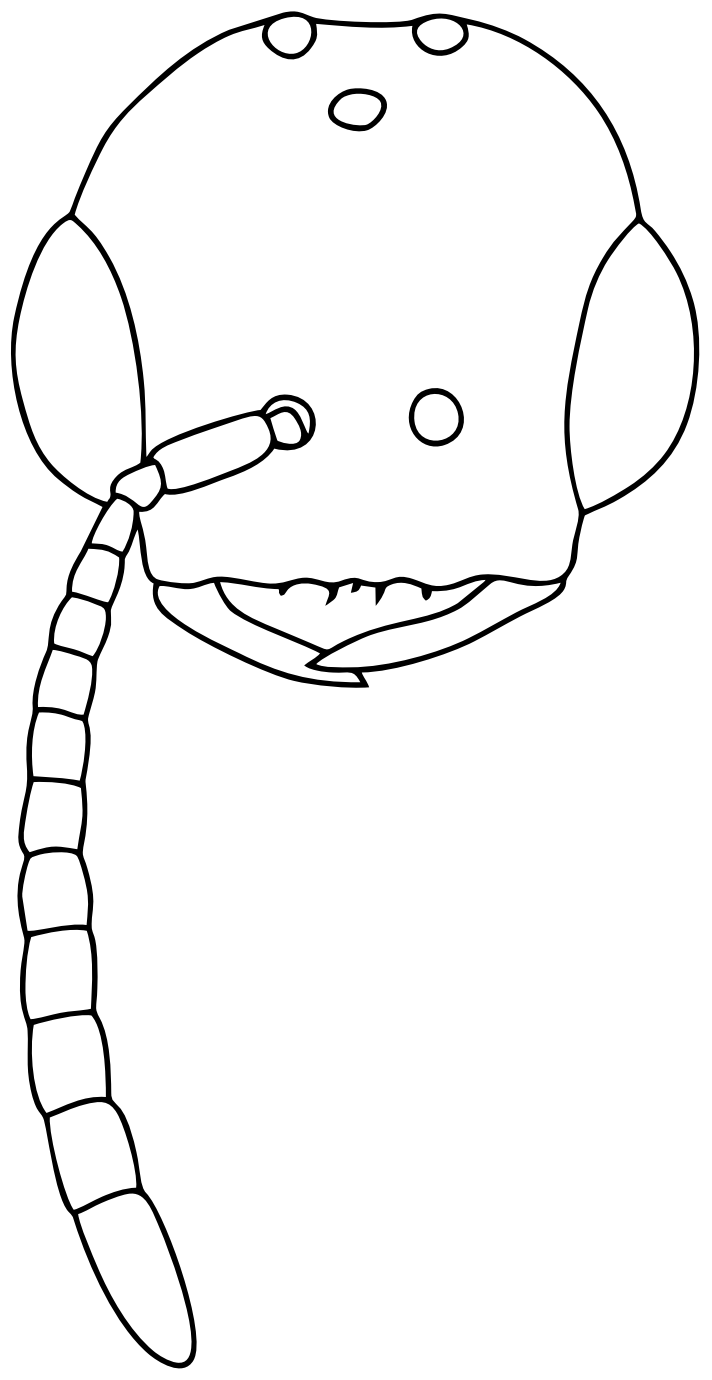

\section{Identificação das espécies neotropicais}

Todas as chaves de identificação para as espécies de Stigmatomma disponíveis até o momento (BROWN, 1960; LATTKE, 1991; LACAU; DELABIE, 2002; ARIAS-PENNA, 2008) ignoram o número correto de segmentos antenais de S. bierigi Santschi, que seria onze, ao invés de doze - informação não mencionada na descrição original da espécie. Estamos preparando uma chave atualizada para as espécies neotropicais de Stigmatomma que além de contornar este problema, é baseada em carácteres com maior valor diagnóstico (ESTEVES; FISHER, em preparação).

\section{Perspectivas}

A delimitação dos gêneros de Amblyoponinae ainda não é perfeita, e dados moleculares indicam que o gênero Stigmatomma é polifilético (FISHER, B.L., dados ainda não publicados). Ainda assim, as espécies neotropicais deste gênero aparentemente fazem parte de uma mesma linhagem evolutiva (FISHER, B.L., dados ainda não publicados); no entanto, $S$. heraldoi e os espécimes de espécies ainda não descritas e presentes em coleções entomológicas (observação pessoal) não foram considerados em tal estudo.

Quanto à taxonomia das espécies de Amblyoponinae, todos os três gêneros neotropicais precisam ser melhor estudados. Paraprionopelta é apenas conhecida por poucos indivíduos de uma mesma casta. Os limites de Prionopelta amabilis, $P$. antillana e $P$. marthae não são claros, e a última revisão do gênero foi realizada por Brown (1960), o que provavelmente indica que numerosos espécimes, e prováveis novas espécies, estejam engavetados em coleções entomológicas. Finalmente, algumas espécies de Stigmatomma precisam de descrições mais detalhadas para que caracteres variáveis, como tamanho, impressão de escultura e números de dentes mandibulares e setas clipeais, sejam substituídos por caracteres de maior valor diagnóstico (observações pessoais).

Claramente, um dos maiores empecilhos para o conhecimento da biologia e taxonomia de Amblyoponinae é o hábito hipogeico de seus membros. A grande maioria dos espécimes presentes em coleções entomológicas foram coletados através de técnicas quantitativas que não acessam apropriadamente o microhabitat onde essas formigas nidificam. Dessa forma, quando coletadas, 
as espécies de Amblyoponinae são representadas por um ou poucos indivíduos, impedindo o avanço dos estudos sobre essa subfamília de formigas: a variação morfológica existente dentro das colônias e entre elas não pode ser avaliada adequadamente; machos raramente são associados com operárias de uma mesma colônia, o que dificulta a preparação de chaves de identificação para essa casta; e a biologia reprodutiva e alimentar são desconhecidas ou são generalizadas por poucas observações de pouquíssimas espécies. Assim, sugerimos que mirmecólogos dediquem mais tempo à coleta manual de formigas, principalmente no interior do solo e em troncos podres, e à história natural. Essa sugestão não se aplica apenas a Amblyoponinae, mas a qualquer outro taxon de formiga que possua hábitos hipogeicos.

\section{Referências}

ANTWEB. AntWeb. Disponível em $<$ http://www. antweb.org> (acesso: 10.viii.2014a)

\section{ANTWEB. Antweb: Paraprionopelta}

minima / CASENT0628950. Disponível em

$<$ http://www.antweb.org/specimenImages.

do? name $=$ CASENT0628950 $>$ (acesso: 10.viii.2014b)

ARIAS-PENNA, T. M. Subfamilia Amblyoponinae. In: Jiménez, E.; Fernández, F.; Arias, T.M.; LozanoZambrano, F.H. (eds.). Sistemática, biogeografía y conservación de las hormigas cazadoras de Colombia. Bogotá: Instituto de Investigación de Recursos Biológicos Alexander von Humboldt. 2008. p. 41-51

BILLEN, J.; THYS, B.; ITO, F.; GOBIN, B. The pretarsal footprint gland of the ant Amblyopone reclinata (Hymenoptera, Formicidae) and its role in nestmate recruitment. Arthropod Structure and Development, v. 34, p. 111-116, 2005.

BOLTON, B. An online catalog of the ants of the world. Disponível em $<$ http://antcat.org $>$ (acesso: 10.viii.2014)

BOLTON, B. Synopsis and classification of Formicidae. Memoirs of the American Entomological Institute. 2003. Vol. 71. The American Entomological Institute, Gainesville, FL.

BORGMEIER, T. Myrmecologische Studien, I. Anais da Academia Brasileira de Ciencias, v. 29, p. 103-128, 1957.

BORGMEIER, T. Formigas novas ou pouco conhecidas de Costa Rica e da Argentina (Hymenoptera, Formicidae). Revista Brasileira de Biologia, v. 9, p. 201-210, 1949.
BRADY, S. G.; FISHER, B. L.; SCHULTZ, T. R.; WARD, P. S. The rise of the army ants: Diversification of the specialized predatory doryline ants. BMC Evolutionary Biology, v.14, p. e93, 2014.

BRADY, S. G.; SCHULTZ, T. R.; FISHER, B. L.; WARD, P. S. Evaluating alternative hypotheses for the early evolution and diversification of ants. Proceedings of the National Academy of Sciences of the United States of America, v.103, p. 18172-18177, 2006.

BROWN, W.L., Jr. Concoctio genus nov. Pilot Register of Zoology, n. 29, p. 1, 1974.

BROWN, W. L., JR.; GOTWALD, W. H., JR.; LÉVIEUX, J. A new genus of ponerine ants from West Africa (Hymenoptera: Formicidae) with ecological notes. Psyche, v. 77, p. 259-275, 1971.

BROWN, W. L., Jr. Contributions toward a reclassification of the Formicidae. III. Tribe Amblyoponini (Hymenoptera). Bulletin of the Museum of Comparative Zoology, v. 122, p. 143-230, 1960.

EMERY, C. Descriptions de quelques fourmis nouvelles d'Australie. Annales de la Société Entomologique de Belgique, v. 39, p. 345-358, 1895.

ERICHSON, W. F. Beitrag zur Insecten-Fauna von Vandiemensland, mit besonderer Berücksichtigung der geographischen Verbreitung der Insecten. Archiv für Naturgeschichte, v. 8, n. 1, p. 83-287, 1842.

FOREL, A. Ameisen aus Guatemala usw., Paraguay und Argentinien (Hym.). Deutsche Entomologische Zeitschrift, v. 1909, p. 239-269, 1909.

FOREL, A. Ameisen aus Java. Gesammelt von Prof. Karl Kraepelin 1904. Mitteilungen aus dem Naturhistorischen Museum in Hamburg, v. 22, p. 1-26, 1905.

GOTWALD, W. H., JR.; LÉVIEUX, J. Taxonomy and biology of a new West African ant belonging to the genus Amblyopone (Hymenoptera: Formicidae).

Annals of the Entomological Society of America, v. 65, p. 383-396, 1972.

HOLLDOBLER, B.; OBERMAYER, M.; WILSON, E.O. Communication in the primitive cryptobiotic ant Prionopelta amabilis (Hymenoptera: Formicidae). Journal of Comparative Physiology, v. 170A, p. 9-16, 1992.

HOLLDOBLER, B.; WILSON, E. O. Ecology and behavior of the primitive cryptobiotic ant Prionopelta amabilis (Hymenoptera: Formicidae). Insectes Sociaux, v. 33, p. 45-58, 1986. 
ITO, F. Observation of group recruitment to prey in a primitive ponerine ant, Amblyopone sp. (reclinata group) (Hymenoptera: Formicidae). Insectes Sociaux, v. 40, p. 163-167, 1993a.

ITO, F. Social organization in a primitive ponerine ant: queenless reproduction, dominance hierarchy and functional polygyny in Amblyopone sp. (reclinata group) (Hymenoptera: Formicidae: Ponerinae). Journal of Natural History, v. 27, p. 1315-1324, 1993 b.

ITO, F. Preliminary report on queenless reproduction in a primitive ponerine ant Amblypone sp. (reclinata group) in West Java, Indonesia. Psyche, v. 98, p. 319-322, 1991.

ITO, F.; BILLEN, J. Larval hemolymph feeding and oophagy: behavior of queen and workers in the primitive ponerine ant Prionopelta kraepelini (Hymenoptera, Formicidae). Belgian Journal of Zoology, v. 128, p. 201-209, 1998.

KÜCK, P.; HITA GARCIA, F.; MISOF. B. Improved phylogenetic analyses corroborate a plausible position of Martialis heureka in the ant tree of life. PLoS One, v. 6, p. e21031, 2011.

KUSNEZOV, N. Zwei neue Ameisengattungen aus Tucuman (Argentinien). Zoologischer Anzeiger, v. 154, p. 268-277, 1955.

LACAU, S.; DELABIE, J. H. C. Description de trois nouvelles espèces d'Amblyopone avec quelques notes biogéographiques sur le genre au Brésil (Formicidae, Ponerinae). Bulletin de la Société Entomologique de France, v. 107, p. 33-41, 2002.

LATTKE, J. E. Studies of neotropical Amblyopone Erichson (Hymenoptera: Formicidae). Contributions in Science, v. 428, p. 1-7, 1991.

LUCKY, A.; TRAUTWEIN, M. D.; GUÉNARD, B. S.; WEISER, M. D.; DUNN, R. R. Tracing the rise of ants Out of the ground. PLoS One, v. 8, p. e84012, 2013.

MASUKO, K. Predation of centipedes by the primitive ant Amblyopone silvestrii. Bulletin of the Association of Natural Sciences of Senshu University, v. 24, p. 3543, 1993.

MAYR, G. Südamerikanische Formiciden. Verhandlungen der Kaiserlich-Königlichen Zoologisch-Botanischen Gesellschaft in Wien, v. 37, p. 511-632, 1887.

MAYR, G. Myrmecologische Beiträge. Sitzungsberichte der Kaiserlichen Akademie der Wissenschaften in Wien. Mathematisch-Naturwissenschaftliche Classe. Abteilung I, v. 53, p. 484-517, 1866.
MOREAU, C. S. Inferring ant evolution in the age of molecular data (Hymenoptera: Formicidae). Myrmecological News, v. 12, p. 201-210, 2009.

MOREAU, C. S.; BELL, C. D. Testing the museum versus cradle tropical biological diversity hypothesis: phylogeny, diversification, and ancestral biogeographic range evolution of the ants. Evolution, v. 67, n. 8, p. 2240-2257, 2013.

MOREAU, C.; BELL, C. D.; VILA, R., ARCHIBALD, S. B.; PIERCE, N. E. Phylogeny of the ants: diversification in the age of Angiosperms. Science, v. 312, p. 101-104, 2006.

OUELLETTE, G. D.; FISHER, B. L.; Girman, D.J. Molecular systematics of basal subfamilies of ants using 28S rRNA (Hymenoptera: Formicidae). Molecular Phylogenetics and Evolution, v. 40, p. 359-369, 2006.

PAGLIANO, G.; SCARAMOZZINO, P. Elenco dei generi di Hymenoptera del mundo. Memorie della Società Entomologica Italiana, v. 68, p. 1-210, 1990.

RABELING, C.; BROWN, J. M.; VERHAAGH, M. Newly discovered sister lineage sheds light on early ant evolution. Proceedings of the National Academy of Sciences of the United States of America, v. 105, p. 14913-14917, 2008.

ROGER, J. Einige neue exotische Ameisen-Gattungen und Arten. Berliner Entomologische Zeitschrift, v. 6, p. 233-254, 1862.

ROGER, J. Die Ponera-artigen Ameisen (Schluss). Berliner Entomologische Zeitschrift, v. 5, p. 1-54, 1861.

ROGER, J. Beiträge zur Kenntniss der Ameisenfauna der Mittelmeerländer. I. Berliner Entomologische Zeitschrift, v, 3, p. 225-259, 1859.

SANTSCHI, F. Quelques fourmis de Cuba et du Brésil. Bulletin. Société Entomologique d'Egypte, v. 14, p. 7583, 1930.

SANTSCHI, F. Formicides de l'Afrique occidentale et australe du voyage de Mr. le Professeur F. Silvestri. Bollettino del Laboratorio di Zoologia Generale e Agraria della Reale Scuola Superiore d'Agricoltura. Portici, v. 8, p. 309-385, 1914.

SANTSCHI, F. Quelques fourmis de l'Amérique australe. Revue Suisse de Zoologie, v. 20, p. 519-534, 1912.

SAUX, C.; FISHER, B. L.; SPICER, G. S. Dracula ant phylogeny as inferred by nuclear $28 \mathrm{~S}$ rDNA sequences and implications for ant systematics (Hymenoptera: Formicidae: Amblyoponinae). Molecular Phylogenetics and Evolution, v. 33, p. 457-468, 2004. 
TRANIELLO, J. F. A. Caste in a primitive ant: absence of age polyethism in Amblyopone. Science, v. 202, p. 770-772, 1978.

WARD, P. S. Adetomyrma, an enigmatic new ant genus from Madagascar (Hymenoptera: Formicidae), and its implications for ant phylogeny. Systematic Entomology, v. 19, p. 159-175, 1994.

WARD, P. S.; BRADY, S. G.; FISHER, B. L.; SCHULTZ, T. R. The evolution of myrmicine ants: phylogeny and biogeography of a hyperdiverse ant clade (Hymenoptera: Formicidae). Systematic Entomology. 2014. online versão.

WILSON, E. O.; HÖLLDOBLER, B. The rise of the ants: a phylogenetic and ecological explanation. Proceedings of the National Academy of Sciences of the United States of America, v. 102, p. 7411-7414, 2005.

XU, Z.H. Two new genera of ant subfamilies Dorylinae and Ponerinae (Hymenoptera: Formicidae) from Yunnan, China. Zoological Research, v. 21, p. 297-302, 2000.
YAMANE, S.; BUI, T.V.; EGUCHI, K. Opamyrma hungvuong, a new genus and species of ant related to Apomyrma (Hymenoptera: Formicidae: Amblyoponinae). Zootaxa, v. 1767, p. 55-63, 2008.

YOSHIMURA, M.; FISHER, B.L. A revision of the ant genus Mystrium in the Malagasy region with description of six new species and remarks on Amblyopone and Stigmatomma (Hymenoptera, Formicidae, Amblyoponinae). ZooKeys, v. 394, p. 1-99, 2014.

YOSHIMURA, M.; FISHER, B.L. A revision of male ants of the Malagasy Amblyoponinae (Hymenoptera: Formicidae) with resurrections of the genera Stigmatomma and Xymmer. PLoS One, v. 7, n. 3, p. e33325, 2012. 